\title{
Limaginaire du Bureau et Les Usages du Fétiche au Gabon
}

\author{
Jean-Emery Etoughé-Efé \\ Sociologue Chargé de recherche, \\ BP. IRSH 846 Libreville- Gabon \\ E-mail:efemery78@yahoo.fr \\ $\mathrm{Tel}$ (241) 07.36.06.64
}

\section{Résumé}

En nous appuyant sur les différents imaginaires qui concourent à l'interprétation de certains faits sociaux des peuples du Gabon, il apparaît que le bureau en tant que lieu de travail se nourrit d'un imaginaire. Celui-ci va de l'expression de la promotion sociale à une prétention à l'acquisition du pouvoir. Cet imaginaire semble trouver ses origines dans la période coloniale où le travail du bureau était la fonction des "Blancs" qui maîtrisaient l'écriture alors que le travail manuel était strictement réservé aux populations indigènes. Depuis cette époque, le bureau de travail fait l'objet de toutes les attentions et convoitises. Et dès lors qu'ils sont installés, certains occupants s'efforcent, par tous les moyens, de le conserver. Les stratégies de conservation et de protection contre les agressions diverses sont visibles à travers les usages des fétiches. Ceux-ci sont souvent représentés par certains objets qui ont une importance culturelle sur le plan local. Ces éléments de défense et de protection dans l'espace de travail semblent le transformer en domaine d'appropriation au point de faire du bureau une zone interdite et avoir tendance à le confisquer pour soi.

Mots-clés: Bureau, Domaine, Ecriture, Fétiches, Imaginaire, Pouvoir, Protection.

\section{Introduction}

Un sujet se rapportant à la sociologie du travail peut aussi faire référence aux aspects de la sociologie des religions dans l'explication des phénomènes sociaux qui entourent le monde du travail au Gabon. En nous appuyant sur la sociologie de Weber, lon dira que la compréhension des comportements des individus passe par la prise en compte préalable des structures sociales et des systèmes de valeurs dont les actions sont orientées significativement (Weber 1995:33).

Aussi avons-nous choisi d'étudier l'imaginaire dans le contexte bureaucratique pour montrer son impact sur les comportements des usagers de bureaux au Gabon. 
Le mot imaginaire a, chez ceux quil'emploient, des significations fort différentes, et par certains aspects, il peut donner l'impression d'être une auberge espagnole, ses définitions variant au gré de leurs auteurs. Pour les uns, il est implicitement connoté de façon péjorative, car il s'oppose à la notion de réel, et devient synonyme de chimérique (Thomas I 998: I 5). En effet, l'imagination a le pouvoir de dépasser ce qui est perçu. Grâce à elle, les mondes clos sont transgressés et le visible est outrepassé; elle est à l'origine d'hypothèses et d'inventions concernant des idées, des personnages, des situations qui n'existent pas ou n'ont jamais existé. L'imaginaire est le monde qui n'existe que dans notre imagination. Il est la représentation de ce qui est étranger au réel, de ce qui n'existe pas dans la réalité. Il relève de la fiction, de l'utopie et de l'illusion (Lazorthes r 999:7).

Toutefois, avec le développement des théories, la notion d'imaginaire a pris un tout autre sens. Pour les autres, le terme ne s'oppose plus à celui de réel, il renvoie à un système, à un dynamisme organisateur des images, qui leur confère une profondeur en les reliant entre elles. En effet, dans sa tentative de faire l'esquisse des repères socioculturels de l'expérience de soi en milieu traditionnel en Afrique noire, Sow (1978:11) qualifie d'imaginaire collectif structuré la société des doubles.

Celle qui fonctionne comme la réplique invisible de la société visible des hommes. C'est le lieu de tous les heurts et malheurs, celui des occurrences dramatiques aussi bien que celui à partir duquel se réalisent les succès quotidiens. C'est aussi l'espace au sein duquel se structurent les désirs, les craintes, les angoisses, les espoirs de réussite dans les aléas de la quotidienneté. L'imaginaire n'est donc pas une collection d'images, un corpus, mais un réseau où le sens est dans la relation avec ces images-là.

Cette conception de l'imaginaire est lisible chez K. Marx par sa manière de soutenir le caractère fétichiste de la marchandise dans l'économie capitaliste. Cette esquisse est prolongée par une analyse de l'imaginaire dans la structure institutionnelle de la société moderne (Castoriadis I 975:22 I). Ainsi, au centre de nos préoccupations, il apparaît clairement que l'univers bureaucratique au Gabon est peuplé d'imaginaire d'un bout à l'autre et il y a bien là des systèmes de significations imaginaires qui articulent cet univers bureaucratique à partir de certains indices (Castoriadis I 975:222).

Partant de ces indices, notre objectif, dans cet article, est de montrer l'importance et le sens du bureau dans l'univers socioculturel gabonais à travers l'imaginaire qui l'entoure. Dans la pratique, le bureau se définit, de manière générale, comme une pièce rectangulaire, avec une fenêtre face à la porte, un faux plafond, un éclairage intégré ou apparent suivant le niveau hiérarchique, un bureau (meuble) avec ou sans retour (meubles plus bas placés perpendiculairement), une ou plusieurs armoires et caissons de rangement, quelques chaises, un porte-manteau et une 
corbeille à papiers. Le bureau est toujours, ou presque, face à la porte, dos à la fenêtre (Pélegrin-Genel i 994:20).

Toutefois, le bureau -comme lieu- est devenu le symbole du travail, de son archétype. Il occupe toute la place. C'est le lieu d'identité sociale, le signe d'une organisation hiérarchique, le symbole du pouvoir (Pélegrin-Genel i 994:60). A partir de ce moment, lebureau devient une sorte d'enveloppe aux contours imprécis et, de ce fait, toute irruption dans cet espace sera ressentie par l'occupant voire le propriétaire comme une intrusion. Ainsi, la seule solution pour protéger son intimité ou son intégrité sera de disposer savamment quelques objets personnels qui symbolisent les limites de son jardin secret (Pélegrin-Genel i 994:64).

C'est l'usage de ces objets, qui transformera le bureau en un "domaine"; son domaine à soi tel que défini par J. Favret-Saada (1977:334)1 . Espace public et aussi lieu de représentation du pouvoir, le bureau devient alors une propriété qu'il faut protéger et même défendre. Car il symbolise avant tout le travail et l'identité sociale ensuite. En fait, même s'il est reconnu que "le travail n'est pas seulement un facteur de production indispensable au fonctionnement de l'économie, il est aussi un élément structurant l'identité individuelle et le moyen unanimement reconnu de s'intégrer à la vie sociale" (De Gaulejac r 994:Ior). $\mathrm{Au}$ Gabon, cet élément d'intégration sociale ne s'arrête pas seulement au fait de travailler ou d'avoir un emploi, mais il intègre aussi le fait de disposer d'un bureau car celui-ci est la marque de cet emploi. Aussi, le bureau fait lobjet de convoitises dans un environnement où la compétence ne suffit toujours pas à elle toute seule, “où la moindre réussite ne peut être due qu'à l'effort et où toute autre aisance est suspecte" (Rossatanga-Rignault r 999:89). En effet, "dans un univers où rôde constamment le danger, il n'en coûte rien de suivre certains préceptes traditionnels qui ont fait leurs preuves dans nos sociétés et qui sont susceptibles de prémunir les humains des jeteurs de sorts" (CAmus 2001:68). C'est pourquoi, de nombreuses stratégies sont développées.

Elles vont de la protection, fondée sur les diverses croyances et aussi sur les différentes pratiques religieuses.

Aussi, tout au long de cet article, nous essayerons de décrire quelques faits et comportements qui, au-delà de l'appropriation classique énoncée par Fischer (1983:95) et Pélegrin-Genel (1994:151), révèlent un processus de réappropriation du bureau en tant que « domaine » relevant des attributs propres à un individu.

Le bureau sera défini comme ce que Jeanne Favret-Saada, appelle « domaine » en parlant d'un ensorcelé. En effet, pour cet auteur, le «je » de l'ensorcelé c'est l'ensemble constitué par lui-même et ses possessions, c'est-à-dire l'ensemble qui est socialement rattaché à son nom propre. Dans un tel ensemble, on ne saurait distinguer corps et biens parce que les biens font corps avec celui dont ils portent la marque du nom. 
Le rôle fonctionnel et l'usage de quelques objets permettra de soutenir ce processus. Ce sont ces éléments qui, dans cette contribution, perdront leur aspect premier pour se transformer en fétiches à cause des rôles qu'ils sont appelés à jouer (Tarot 1999:500)2.

Selon De Brosses (Pouillon r 975: I08), le fétiche est un objet matériel. Il peut être aussi bien naturel que fabriqué. Il peut s'agir d'un quelconque objet mais on retrouve généralement des éléments comme l'eau, le sel, le citron, une écorce, etc. L'objet qui sert de fétiche n'est jamais un objet quelconque, choisi arbitrairement, mais il est toujours défini par le code de la magie ou de la religion (Pouillon i 975:I05).

Ainsi, verrons-nous, tout au long de notre cheminement, les fonctions symboliques des différents objets en situation et enfin, mieux percevoir l'appropriation du bureau comme une constitution du domaine. En un mot comment, à partir de l'écriture, se manifeste l'imaginaire du bureau au Gabon.

\section{Lecriture Et Les Changements Sociaux Au Gabon}

En relevant les changements qui ont affecté la structure et les rapports d'ordre politique au Gabon, Balandier (1982:212) souligne que ce sont l'action d'évangélisation et l'enseignement qui ont formé deux types sociaux nouveaux. Il s'agit du chrétien et du lettré.

En ne nous focalisant que sur le lettré, il nous revient que leur action resta longtemps limitée aux centres importants avant d'être ensuite diffusée en raison même du rôle joué par le lettré dans la plupart des villages.

En effet, l'établissement d'un nouvel usage en matière de dot a accru leur influence. La principale innovation a été, ici, de dresser une liste des dons faits à la belle-famille. Il faut aussi ajouter sa qualité d'auxiliaire des chefs, sa fonction d'interprète ou de commentateur des écrits. Autant dire que les signes étaient nombreux pour indiquer le passage d'une civilisation fondée sur la tradition orale à une civilisation sur l'écriture.

Aussi, le recours à la chose écrite ne s'est pas seulement imposé en tant que moyen visant à fonder les innovations coutumières, il s'est imposé aussi en tant que substitut des anciens procédés de preuve.

L'usage de la preuve ne put que s'affirmer dans ces cas. Soit qu'elle est intervenue dans les situations radicalement nouvelles, soit qu'elle a remplacé les symboles matériels autrefois utilisés.

2 Pour Camille Tarot, Le fétiche est le nom donné par les Blancs aux objets de culte et aux pratiques religieuses des peuples et des civilisations de Guinée et d'Afrique occidentale aux XVè et XVIè siècles. Alors que le fétichisme est un nom créé par De Brosses pour désigner toute religion qui a pour objet le culte des animaux ou des être terrestres inanimés. 
C'est là que le besoin d'un appareil bureaucratique simple et en contrecoup et les nouvelles fonctions du lettré de village se sont décelés (Balandier 1982:269).

$\mathrm{Au}$ Congo, par exemple, l'accroissement de la couche sociale des lettrés a été conditionné par la nature de l'économie des Kongo, très anciennement orientée vers l'extérieur et aussi par la proximité des centres et zones d'attraction. Aussi l'emprise de cette couche sociale qu'il est convenu d'appeler "les évolués" s'explique par une tension collective et une volonté de progrès. Elle s'explique aussi et surtout par le fait que les groupements de parenté et les lignages sont restés vivants et capables de créer un champ social à l'intérieur duquel l'individu s'inscrit, même lorsqu'il se trouve éloigné du foyer commun pendant une longue période (Balandier 1982:393). C'est ainsi qu'un transfert a dû être opéré au point de construire tout un imaginaire autour du bureau.

\section{Le Bureau Et Son Imaginaire}

La bureaucratie caractérise l'administration des activités économiques ou politiques par le moyen des règles universelles stables écrites et d'un personnel spécialisé. Malgré les innovations du lexique et la singularité des contextes idéologiques (Akoun et Ansart 1999), la bureaucratie n'est en rien une construction de l'occident moderne.

Le développement qu'on lui connaît fut précédé, en réalité, par une expérience d'au moins deux millénaires dans des civilisations aussi différentes que celle de la Chine ancienne, des empires égyptien, romain et ottoman, et de la cité des Incas dans l'Amérique précolombienne.

Toutefois, la bureaucratie constitue toujours une zone d'incertitude, rebelle à l'analyse scientifique et l'un des terrains d'élection des professions de foi et des mythes idéologiques de notre temps (Crozier 1963:216) Selon le sociologue Thierry Pillon, "le bureau, aujourd'hui, est un monde hétérogène où coexistent encore le bureau traditionnel de type administratif, et le chic débraillé qui prévaut par exemple chez les publicitaires"3.

Toutefois, la conception du bureau au Gabon relève d'un ensemble de situations et de représentations liées à la définition qu'en donne Max Weber (1995:299), d'une part, et à la situation coloniale qu'a connue ce pays, d'autre part.

En effet, pour Max Weber, l'administration bureaucratique est synonyme de domination en vertu du savoir; c'est en cela que son caractère est spécifiquement rationnel. Par delà l'énorme puissance que détermine le savoir spécialisé, la bureaucratie a tendance à accroître davantage encore sa puissance par le savoir du service.

3 Interview accordée au journal « Ouest France » du 2 mai 2001. 
Au sortir des années de colonisation, grâce à l'acquisition d'un savoir nouveau qui valorise la connaissance écrite, celle du lettré ou du bureaucrate, aux dépens de la connaissance orale (Balandier i 965:35), le bureau va supplanter les "plantations" et marquer la différence avec le travail ouvrier dans les chantiers forestiers. Aujourd'hui, par exemple, l'employé bureaucrate constitue un élément pertinent pour cerner la situation des employés qui travaillent dans les administrations.

A ce sujet, le sociologue G. Balandier (1954:507) relève par exemple que, parmi les travailleurs brazzavillois, l'emploi de manœuvre, était reconnu par la presque totalité des travailleurs comme "le plus mauvais", parce qu'il exigeait un travail pénible, rapportant les salaires les plus bas et n'entraînant aucune considération. Ainsi les professions le plus attirantes étaient celles qui correspondaient aux techniques nouvelles apportées par les Européens et notamment celles liées à l'écriture (Goody 1994:87)4-1'administration et l'enseignement principalementpar le prestige qu'ils procurent. Il semble bien que l'écriture ait établi une hiérarchisation des catégories socioprofessionnelles (Erny 1999:158)5. Dans ce contexte, on dirait même que l'histoire coloniale du travail conduit inévitablement au bureau. En effet, au premier rang des cadres locaux indigènes de l'Etat colonial, il y avait les écrivains-interprètes. De 1914 à 1938, ces derniers étaient recrutés et organisés pour les besoins des bureaux du Gouverneur général, des lieutenantsgouverneurs et des chefs de circonscription. Ils étaient destinés à seconder les fonctionnaires européens d'administration générale dans les bureaux des chefslieu des colonies et dans les circonscriptions.

Â partir de 1938, ces cadres indigènes seront recrutés par voie de concours ouverts aux candidats titulaires d'un certificat d'études primaires de l'enseignement public, aux agents titulaires de grades locaux indigènes et aux écrivains, comptables ou dactylographes auxiliaires comptant trois ans de services dans l'administration à l'époque du concours.

Ils étaient nommés et promus par décisions des Lieutenants-Gouverneurs, soumises à l'approbation du Gouverneur Général (Ndombi 1995:95).

Le bureau devient alors l'endroit où l'on affirme sa catégorie sociale ou mieux son pouvoir (Crozier 1963:202).

C'est ainsi que les individus s'approprient, par la force de l'imaginaire des identités valorisées grâce à la magie de l'écriture.

4 L'invention de l'écriture aux alentours de 3000 avant J.C. ne fournit pas seulement un admirable instrument d'engrangement, de précision et d'analyse conceptuelle, amenant des changements révolutionnaires dans la culture, mais permit l'émergence d'une classe de lettrés spécialisés dans la technique et l'art difficiles de l'écriture et dans la façon de regarder les choses, approche intellectuelle de la réalité.

5 Selon Pierre Erny, «certains métiers émergeaient et attiraient tous les regards : ils étaient de ce fait de principaux indicateurs de ce qui était valorisé dans l'univers mental du jeune ». 
En remontant l'histoire, en effet, il apparaît que, dans les premiers siècles des histoires égyptienne, grecque, romaine et chinoise, l'écriture reste un art que possèdent seuls, quelques professionnels hyper privilégiés et auréolés d'un prestige quasi sacré. On ne confiera donc pendant longtemps aux scribes que la transcription d'éléments religieux ou, au plus, intéressant l'exercice du pouvoir (Gaurier et Hesse i 987:75). En réalité, le bureau, dans notre contexte, comme le souligne, J. Tonda (2002:30), répond à la logique des rapports de connaissances constitutifs des relations de commandement et /ou de pouvoir surnaturel $\mathrm{du}$ «Souverain moderne » en colonie et en post colonie. Pour ce dernier, commander ${ }^{6}$ confère le droit d'exiger, de contraindre, d'interdire, de punir, de récompenser, d'être obéi, bref, d'enjoindre ou de diriger, alors que le Souverain moderne s'appelle la Science, la Technique, l'Etat, le Capital, le Dieu chrétien et le Génie sorcier (Tonda 2002:27).

$\mathrm{Au}$ Gabon, le bureau est synonyme d'aisance et signe de richesse. Et si l'argent est le symbole du pouvoir d'une catégorie de personnes, le bureau l'est aussi, par ailleurs. Comme tout pouvoir, le bureau suscite l'envie et la jalousie, il attire "la mauvaise langue" et le "mauvais œil" (Fassin 1992:321). Il semble que ce soient les craintes de la concurrence, de la vulnérabilité et de la déstabilisation dans son emploi qui entraînent les gens à s'abandonner à l'usage du fétiche.

A y regarder de près, l'argent est le premier élément pour lequel l'on utilise le fétiche et relègue les autres avantages au second plan car c'est par lui que l'on peut se procurer les produits du génie scientifique que sont voitures, avions, appareils de toutes sortes, téléphones portables, toutes ces choses extraordinaires qui suscitent, frustrations, haines familiales ou de classe, désirs de mort de l'autre possédant (Tonda 2000:56).

Le bureau, à travers l'imaginaire qu'il nourrit, est assimilé à l'argent, au gain que l'appropriation de ce lieu peut procurer dans l'imaginaire collectif (Mary 1987:142) ${ }^{7}$.

En l'occupant, il faudra, entre autres remparts, s'assurer d'une sécurité personnelle de l'emploi et de la promotion. Car quoiqu' inscrit dans la modernité, il y a toujours un imaginaire qui justifie l'usage du fétiche. Notre société ne faitelle pas partie d'une modernité propre à l'Afrique où des éléments traditionnels s'articulent avec des perceptions plutôt modernes?

6 Âce sujet, nous avons retenu le conflit de compétence relatif à l'époque de la colonisation entre un commandant de cercle et un chef indigène en Haute-Volta rapportée par Amadou Hampâté Bâ, Oui mon commandant! Mémoires II, Arles, Actes Sud, 1994, pp188-198.

7 Le terme d'imaginaire est ici compris au sens que lui donne C.Castoriadis, c'est-à-dire comme un magma de significations sociales véhiculées par des images ou des symboles produits par la faculté originaire de poser ou de se donner, sous le mode de la représentation, une chose et une relation qui ne sont pas données dans la perception. 
En observant tout l'imaginaire qui se construit à travers le bureau, nous constatons une ambivalence de comportements liée à son occupation. En effet, l'espace de travail appelé bureau ${ }^{8}$, comme l'ensemble des éléments qui accompagnent la vie d'un individu dans son milieu socioprofessionnel, fait partie de la relation que l'homme entretient avec les esprits. Il y passe, en théorie, un minimum de huit heures par jour de travail (Pélegrin-Genel 1994:35) ${ }^{9}$.

De plus, c'est l'endroit où l'on reçoit des tiers, sans que cela ne débouche absolument sur des relations particulières. C'est, par excellence, le lieu symbolique à travers lequel s'exerce une suite de luttes entre forces qui sont aux yeux des gabonais des puissances invisibles.

Car, comme le souligne A. Marie, "la réalité africaine contemporaine se caractérise par la précarisation et par la problématisation des solidarités communautaires, dans un climat de contradictions et de tensions accrues qui viennent s'accuser dans l'imaginaire de la sorcellerie et qui engendrent un processus généralisé d'individualisation subjective"(Tonda 2000:56).

Les données de terrain, analysés plus loin, contribuent à conforter cette hypothèse.

\section{Les "Fétiches"}

Les éléments qui ont concouru à cette réflexion ont été recueillis dans diverses localités du pays. Aussi, l'analyse qui suivra reposera t-elle sur l'ensemble des bureaux au Gabon.

En effet, pendant plusieurs mois ${ }^{10}$, nous avons eu l'opportunité d'être reçus dans les bureaux des administrations publiques et privées, dans le cadre des études de différents projets de développement.

Parmi ces éléments, nous avons retenu des fruits de citron exposés ou dissimulés, du sel dans des bibelots sur lesquels sont plantées quelques fleurs artificielles, des chapelets, l'eau bénite, les Bibles, etc., posées dans un tiroir ou dans un coin du bureau.

Pour ces objets qui ne font pas partie des décors conventionnels, quelle définition leur donner, si ce ne sont pas les fétiches?

8 Nous entendons par bureau, une salle pourvue du mobilier nécessaire aux travaux de secrétariat, ou dans laquelle travaillent et reçoivent les membres de l'administration d'une entreprise ou d'une collectivité (bureau du Directeur, de Maire, etc....). c'est aussi un groupe de membres d'une administration, d'une association.

9 Les bureaux du XIXè siècle de l'Administration. Lieux de travail au départ, ils abritaient de fait, toute une vie parallèle. On venait y passer 8 heures, mais on s'y faisait raser, coiffer, on s'y changeait, on y mangeait, on écrivait des vaudevilles, on dessinait des caricatures.

10 De juillet 2000 à mai 2002, dans le cadre de nos activités en tant que consultant pour les projets de développement au Gabon nous avons pu observer, interroger et analyser ce phénomène. 
Il apparaît, en effet, que ces différents fétiches ont tous un sens et leur vertu est d'éloigner ou de neutraliser les mauvais esprits.

Aussi, les différents entretiens personnalisés, à partir des éléments retenus plus haut, nous ont permis d'analyser le phénomène du fétiche à partir de sa capacité ou de sa vertu à rendre son univers de travail propice à soi en toutes circonstances.

Les données quantitatives qui vont suivre dans cette partie relèvent des enquêtes de terrain par questionnaires et guides d'entretien sur un échantillon mixte composé de 139 personnes dont la particularité est de travailler dans un bureau et d'avoir une expérience professionnelle de plus de cinq années ${ }^{11}$.

L'analyse de ces données montre que la plupart des bureaucrates interrogés croient en Dieu, mais en même temps ces personnes utilisent les différents fétiches pour se protéger des mauvais esprits et les maladies mystiques et autres envoûtements qui peuvent perturber la sérénité dans leur emploi de bureaucrate.

Toutefois, pour une meilleure lisibilité, nous avons choisi de différencier les hommes des femmes car il apparaît dans les différentes données que, par endroits, les femmes sont plus enclines à certaines pratiques fétichistes de protection que les hommes.

En effet, selon les informations recueillies chez les femmes, la sorcellerie au bureau se manifeste à travers les maladies "mystiques", les envoûtements et les blocages au travail. Cette sorcellerie est interprétée au travers des envoûtements et des morts mystérieuses (50,75 pour cent) d'une part, d'autre part, il y a le phénomène du vampire et du "fusil nocturne" selon 13,43 pour cent des femmes.

Pour 23,08 pour cent, elles auraient été victimes de la sorcellerie à travers des herbes dans les toilettes du bureau, devant la porte ou par des maladies mystérieuses.

Il apparaît que ce sont les mêmes manifestations qui sont constatées chez les hommes. Ces derniers relèvent aussi des objets "mystiques" pour justifier les preuves de la sorcellerie dans leur bureau.

Ainsi, pour 29,41 pour cent des hommes, la présence de ces objets supposés contenir les herbes, les plumes ou les poudres diverses incitent à la prudence.

Selon 11,76 pour cent d'entre eux, les initiations aux religions traditionnelles et les maladies que nont pu traiter que les tradipraticiens autrement appelés les "nganga" témoignent de la réalité de ces manifestations.

11 Nous avons interrogé 139 personnes (50 femmes et 79 hommes) pendant le mois de janvier 2004 à Libreville. Malgré les réticences des enquêtés à reconnaître les usages des fétiches dans les bureaux, les pourcentages retenus sont ceux supérieurs ou égaux à 10 pour cent pour une meilleure lecture d'un phénomène qui relève encore $d u$ secret. 
Par ailleurs, à la question contre quoi pensez-vous vous protéger? 31,25 pour cent des femmes évoquent la lutte contre les mauvais esprits et les fétiches des autres, le Diable et les péchés $(27,08$ pour cent ) et enfin le pouvoir nuisible des sorciers (12,5 pour cent ). Les hommes aussi se protègent contre les mauvais esprits dans 24,36 pour cent des cas, contre les mauvais sorts et les fétiches (19,32 pour cent ), les sorciers et les méchants (11,54 pour cent ) et enfin contre les attaques mystiques selon 10,26 pour cent des personnes interrogées.

Les types de protections sont très variés ; pour les femmes, les principales protections sont l'eau bénite qu'elles aspergent dans les coins du bureau (33,33 pour cent ), le chapelet qu'elles accrochent dans un coin de la pièce $(24,24$ pour cent ), la Bible dans un angle du meuble de bureau (18,18 pour cent ) et le sel dans un pot servant au décor (18,18 pour cent ). Alors que pour 56,82 pour cent des hommes, la prière est la protection essentielle. Les autres protections nont pas recueilli un pourcentage supérieur ou égal à 10 pour cent, à l'exemple de l'initiation au Bwiti (09, 09 pour cent ) le Biéri ou Melan (4,54 pour cent ), les scarifications (4,54 pour cent), etc. Ces faibles pourcentages peuvent laisser croire à une faiblesse des religions traditionnelles, mais ceux-ci restent à nuancer par le fait que les gens n'avouent presque jamais publiquement leurs pratiques religieuses de cet ordre. La peur d'être indexé de sorcier ou de féticheur en est une des raisons. Il semble, effectivement que l'urbanisation dans la vie des Africains engendre une distanciation par rapport aux rites traditionnels. Quelque soit son statut professionnel, la situation urbaine du gabonais lui impose des choix religieux en même temps quelle limite sa pratique de certaines croyances (Ngoua-Nguema 1998:35).

En ce qui concerne les matériaux et objets qui servent régulièrement de protection chez les femmes, ce sont la Bible (20 pour cent ), le citron (10 pour cent ), l'eau bénite (26 pour cent ), le crucifix (16 pour cent ), le chapelet (16 pour cent ) et le sel (12 pour cent ). Chez les hommes, ces objets sont assimilés aux gourmettes, aux bagues, aux chaînettes $(15,52$ pour cent ) et à la Bible $(24,14$ pour cent ). L'eau bénite, le sel et le citron sont utilisés par 18,96 pour cent, de femmes alors que le chapelet concerne 13,79 pour cent des hommes interrogés. Le piment sert de fétiche à 10,34 pour cent d'hommes.

Ces fétiches ont pour fonction chez les femmes de contrer les attaques mystiques (42, 86 pour cent ), de purifier les lieux où ces objets sont placés (14,28 pour cent ), d'anéantir les forces négatives (14, 28 pour cent ), de briser les mauvais sorts $(14,28$ pour cent $)$ et d'éloigner les mauvais esprits du bureau $(14,28$ pour cent $)$. Chez les hommes par contre, ces objets anéantissent les forces négatives (40,74 pour cent), épargnent des mauvais visiteurs (22, 22 pour cent ), protègent de tout $(22,22$ pour cent $)$, et purifient les lieux où ils sont placés dans le bureau (11,11 pour cent ). 
Les aspects relatifs aux choix et emplacements des protections varient selon les sexes. Les femmes avancent l'argument de Dieu qui est la plus grande protection (51,85 pour cent ). En effet, il ressort des résultats des enquêtes que pour les femmes, leurs objets de protection sont placés à l'entrée du bureau $(24,24$ pour cent ), dans le sac (24,24 pour cent ), dans le tiroir (21,21 pour cent ), et sur les murs pour ce qui concerne les versets bibliques (12 pour cent ).

Chez les hommes, la croyance en Dieu est la protection la plus sûre selon 35,85 pour cent d'hommes, les autres protections étant inefficaces à leurs yeux $(13,21$ pour cent ). D'autres utilisent un fétiche qui leur a été conseillé par un tiers. Cette catégorie représente 11,32 pour cent des hommes de notre échantillon. Les différents objets recensés sont placés à divers endroits du bureau ou sur soi, c'est le cas du citron. Les hommes choisissent aussi la porte ou la fenêtre (32 pour cent ), le tiroir (16 pour cent ) et même le fauteuil sur lequel ils sont assis pour assurer leur protection (12 pour cent).

Au delà de la différence de sexe, toutes ces stratégies ont pour but d'échapper à toutes les tentatives de déstabilisation dans le bureau. En effet, et au regard des réponses recueillies, il ressort que 60 pour cent des femmes se protégent contre leurs ennemies. Une autre raison réside dans le fait qu'elles s'y investissent pour ne pas perdre leur emploi (25 pour cent ). Une minorité veut défier la concurrence des autres (10 pour cent ).

Les hommes, quant à eux, utilisent les fétiches pour bloquer l'action des ennemis (29,27 pour cent ). Par ailleurs, ils veulent lutter contre les mauvais esprits (17,07 pour cent ). Dans une moindre mesure, leur objectif est de faire face à la menace sorcellaire et mystique de leur entourage (12,19 pour cent ). Ils tentent aussi de se protéger et surtout de sauvegarder leur emploi (19,51 pour cent ) et enfin la prudence recommande de se protéger dans un environnement aussi peu sûr que celui du travail (12,19 pour cent ).

Après avoir recueilli les motivations des différents individus, nous nous intéresserons maintenant aux fonctions symboliques du fétiche au bureau afin de mieux appréhender l'évolution du phénomène dans la société bureaucratique gabonaise. Ceci nous permettra de mieux exposer les motivations qui soustendent l'usage du fétiche.

\section{Les Fonctions Symboliques Du Fétiche}

L'imaginaire doit utiliser le symbole, non seulement pour s'exprimer, mais pour exister, pour passer du virtuel à quoi que ce soit de plus (Castoriadis I 975:I77). Comme le souligne Louis-Vincent Thomas (1995:107), le symbole est l'association d'une image avec une idée abstraite. Aussi, l'usage du symbole 
est, en réalité, une mise en œuvre de l'abstraction, c'est-à-dire une démarche vers une codification générale de toute expression. En effet les symboles sont des systèmes de représentations, à degré démographique très faible, mais jamais nuls. Aussi pour interpréter quelques symboles, nous allons adopter la position mixte que propose Gilbert Durand (1992:38) quand il nous parle de trajet anthropologique, c'est-à-dire l'incessant échange qui existe entre les pulsions subjectives et assimilatrices et les intimations objectives du milieu cosmique et social. Ainsi, le bureau, à son tour devient une forêt de symboles fondé sur la répartition et la fonction de la pièce, de la décoration, de certaines parties telles que la porte, le secrétaire, l'armoire, etc. Avec les symboles, nous abordons les formes les plus riches de l'imaginaire, ce qu'il est convenu d'appeler notre imaginal (Durand 1993:9).

Considéré par les historiens des religions comme le premier degré du développement de l'esprit religieux dans l'humanité, le fétiche, qui s'appliquait d'abord au culte des peuples noirs d'Afrique, convient également à l'état religieux de tous les peuples qui attribuent à certains objets vivants ou inanimés de la nature, un pouvoir magique ${ }^{12}$.

Il semble donc que la présence du fétiche dans le bureau relève du procès de cultures qui se traduit dans la conception dualiste et conflictuelle du monde moderne par la société gabonaise. Cette société se trouve placée aux confins de la modernité et de la tradition.

Le bureau étant une structure moderne, et si la pratique du fétiche procédait uniquement de la volonté de briser la carrière de quelqu'un à qui on voue une jalousie, alors pour prévenir ce "danger", la personne menacée entreprend des démarches propitiatoires ${ }^{13}$ en s'abritant derrière le pouvoir du fétiche. Il se sert, ainsi, d'une sorte de bouclier qui le protègerait de toute offence sorcière. Le fétiche, à travers ses différents usages, joue effectivement un rôle social à travers la réalité symbolique qu'il manifeste dans les différentes situations des agents de bureau au Gabon. Et c'est pourquoi ces derniers vont jusqu'à s'approprier ces espaces au point de constituer des domaines propres.

12 Aujourd'hui comme hier, les sociétés contemporaines éprouvent toujours le besoin impérieux de se prémunir contre tout et par tous les moyens. Et les individus sont constamment invités à recourir à diverses techniques d'autodéfense psychologiques capables de leur fournir un halo protecteur contre toutes formes d'agression. Ainsi, un coureur de rallye à moto déclarait le 29 décembre 2002 sur la radio «France-Info » que l'élément le plus important de sa moto était le lapin en peluche fixé au guidon et qui allait attaquer avec lui son quatrième «Paris-Dakar».

13 Â travers son film, sur la République Démocratique du Congo (RDC), intitulé : " l'Afrique en morceaux. La tragédie des grands lacs », le réalisateur Jihan El Tahri montre bien ce que c'est que le propitiatoire. En effet, dans ce document, lors d'une passation de service entre deux officiers supérieurs (rwandais et congolais), le nouvel arrivant a amené une chèvre qu'il a égorgée et aspergé son sang partout dans le bureau, sur l'échelle, sur les escaliers, au sol pour chasser les démons rwandais ». Voir Canal+/ CAPA Production/Plani-Presse 2001. 


\section{La Constitution Du “Domaine”.}

Pour aborder la constitution du domaine, nous partons de la notion d'appropriation $^{14}$ sous les deux angles juridique et technique pour aboutir à un effet psychologique ayant une portée sociologique. Ceci, dans la mesure où cette appropriation se manifeste comme l'exercice d'une autorité, d'un contrôle ou d'un pouvoir. Le contrôle psychologique de l'espace se réfère ici à l'idée d'espace personnel. Ce contrôle de l'espace du bureau passe par un ensemble d'astuces mis en évidence pour s'approprier ce lieu. Notons, par ailleurs, que l'idée d'appropriation désigne globalement l'acte de prendre quelque chose pour soi, ou l'acte de rendre quelque chose propre à son usage. L'appropriation inclut, pour ainsi dire, un ensemble de moyens d'actions ou de comportements à travers lesquels s'opère une structuration de l'espace et une re-disposition des choses et des objets. L'appropriation peut se définir comme l'ensemble des pratiques exercées sur l'espace en réponse aux besoins explicites de contrôle (Fischer I 983:95) et de pouvoir. Le bureau, mieux que le domicile est mis en valeur pour des raisons de prestige personnel. Lon y reçoit ses parents et ses amis pour exposer la réussite professionnelle. L'individu s'approprie son environnement pour définir son identité; il se crée ainsi une marge personnelle d'autonomie (Bernoux I98 I:I4I).

C'est, donc, par la possession du bureau que le bureaucrate devient un cadre (Ferréol 2000:98) ${ }^{15}$. Pour certaines personnes qui se font appeler ainsi au Gabon $^{16}$, ce terme révèle leur fonction d'employé dans un bureau à un niveau de rémunération et à la possession d'un certain nombre de signes, supposés de richesse.

Car s'il est reconnu que l'attachement très fort au titre de cadre, que manifestent, notamment les agents de bureau, fonctionne comme un leurre, c'est néanmoins un leurre efficace (Gresle, Perrin, Panoff et Tripier I 990) parce qu'il se fonde sur un ensemble de représentations de pouvoir ${ }^{17}$ qui conduisent inexorablement vers une confiscation sous forme d'appropriation de l'espace de travail.

14 La notion d'appropriation peut être définie sous plusieurs angles: du point de vue juridique, d'un point de vue technique et dans une perspective psychologique plus large.

15 Au Gabon, le terme de "cadre » désigne, au Gabon les actifs dont les niveaux de diplômes sont élevés. Très souvent ces hautes qualifications ne s'accompagnent pas de hauts niveaux de revenus.

16 L'ensemble des ressortissants de la province de l'Ogooué-Ivindo au nord-est du Gabon ayant un niveau socioprofessionnel moyen ou élevé se servent du terme "cadre " pour s'appeler entre eux. Ces différents cadres se retrouvent pour des manifestations précises comme les parties de Foot-Ball les dimanches ou les autres manifestations associatives.

17 Pendant de nombreuses années, à Ndjolé, dans la région du Moyen-Ogooué, les hauts fonctionnaires ayant le diplôme de "docteur » issus de cette préfecture forment un groupe de cadres dans lequel ils n'acceptent aucune intrusion d'un moins diplômé qu'eux. 
Personne n'a le droit d'y accéder, par exemple, en cas d'absence parce que le bureau devient une propriété à l'intérieur de laquelle on a disposé des "petites choses" particulières. Une sculpture de sa région, les dessins d'école des enfants, un objet-souvenir, des pots de fleurs. Les photographies, les souvenirs de voyages et les présents sont les éléments les plus courants de l'appropriation. Toutefois, au Gabon, le bureau est aussi le prolongement de l'individu dans un système de représentations sociales, c'est-à-dire sa vie. Son corps est indissociable de son esprit, de son entité supérieure. De ce fait, au-delà du phénomène d'appropriationpersonnalisation qui est quasi universel, il faut souligner que la constitution du domaine garantit aux bureaucrates la sécurité, l'équilibre socioprofessionnel, l'équilibre spirituel, l'assurance d'être préservé d'un licenciement et la résistance aux agressions diverses et variées.

\section{Le Bureau : Un Espace Public Confisqué}

L'exposé de quelques tranches de vie ont été nécessaires pour l'illustration des aspects qui peuvent concourir, sans les justifier, à la compréhension des mobiles qui favorisent la "confiscation" des bureaux.

“...Un jour, je suis parti au travail le matin. Mais l'après-midi, je n'ai pas pu aller. Il y avait des problèmes à la maison entre ma sœur et son mari.

Ils se sont bagarrés au moment où je me préparais à aller au boulot. Il y eu des coups et blessures.

Alors, je ne me suis pas rendu au boulot ce jour-là. Ce n'est que le lendemain que je me suis présenté à mon lieu de travail. Mon chef de service m'a déposé une demande d'explications. Je lui ai expliqué ce qui s'était passé.

Il n'a pas voulu comprendre. Il m'a dit: "Monsieur, vous devez remplir la demande d'explications sans quoi, je fais partir un rapport à la direction. J'ai catégoriquement dit non, je ne peux pas, je vous ai déjà expliqué ce qui s'est passé. Et finalement, il a pris sa décision. Il est parti voir le directeur d'agence. Il lui a expliqué ce qui se passait. Le directeur s'est présenté, il m’a appelé et m’a dit : à partir d'aujourd'hui, vous avez une mise à pied d'un mois...”.

“... J'ai fait état de ce qui m’arrivait à ma mère. Elle a trouvé que ce n'est pas normal. C'est un système qui s'est passé mystiquement. C'est ton propre collègue qui est à l'origine de ton malheur".

"Ầ mon insu, il est rentré dans mon bureau et il a pris le stylo-encre avec lequel je travaillais. Il est parti avec, voir les féticheurs. Ensuite, il est revenu le déposer. Mon sort était scellé. Alors, tout ce que j'écrivais, quand ça partait, tout le monde voyait ça flou au niveau de la direction. On doutait désormais de mon travail. Donc il y a des gens qui font en sorte qu'on vous enlève du travail, ils passent 
par une piste obscure. C'est à ce moment que j'ai décidé d'aller voir un féticheur pour qu'il blinde mon bureau dès que j'ai repris le travail. J'ai un petit nœud de tissus noir et rouge quelque part dans mon bureau. Et plus personne ne pourra plus s'attaquer à moi aussi facilement" ${ }^{18}$.

Dans le même sens, un autre poursuit : “...Quand je travaillais à Mouila ${ }^{19}$, j'avais un jour trouvé à l'entrée de mon bureau des tâches de sang.

Cela était mystérieux pour tout le monde. J'avais voulu négliger, mais quelques semaines après j'étais tombé malade. J'avais tout de suite demandé ma mutation sur Libreville" ${ }^{20}$.

"Lorsque j'étais à la RTG chaîne 2, dit ce dernier, je m'enfermais dans mon bureau, dès que j'arrivais pour une séance de travail tous les matins.

Parce que mon entourage m'avait averti de graves problèmes de mysticisme qui y existaient. J'aspergeais mon bureau d'eau bénite et parfois avec de l'huile sacrée qu'un prêtre exorciste m'avait offert" ${ }^{\prime 1}$.

"Je travaille depuis quinze ans et j'ai perdu mon collègue, il y a quatre ans. Selon sa femme, il avait été piégé par son prédécesseur qui ne voulait pas perdre son poste.

Cette vérité avait été révélée par trois nganga.” ${ }^{22}$.

En analysant ces extraits de récits, il apparaît que la perte du bureau constitue une hantise pour toutes les catégories socioprofessionnelles du Gabon. Ainsi, tous les niveaux de la société employée sont représentés.

Devant les menaces que font planer les exigences de la flexibilité au travail, les agressions extérieures au milieu du travail, de la rentabilité de ce dernier et toutes autres agressions de différentes natures, les recours sont multiples dans une société qui se nourrit de toutes ces incertitudes dans un univers social de plus en plus individualisant.

Autrefois protectrice, la société moderne individualise.

Devant les incertitudes de cette individualisation, des stratégies de sécurité et de défense se développent.

C'est ainsi qu'en se référant à la relation que l'employé entretient, désormais, avec son bureau, diverses précautions sont prises pour surmonter les aléas que nous impose la société moderne.

C'est alors que le fétiche, support d'une réalité symbolique de protection et de défense, intervient, sous la forme d'un objet ou d'une prière, pour rassurer les bureaucrates dans leur univers de travail.

18 Extrait du récit de Jean-Louis B., employé de banque à Libreville.

19 Mouila est la capitale provinciale de la Ngounié.

20 Député à l'Assemblée Nationale

21 Ancien Directeur Général RadioTélévision Gabonaise, Chaîne2.

22 Fonctionnaire du ministère du travail 


\section{Conclusion}

Comme nous venons de le voir, les agents de bureau font grand usage d'objets censés renfermer une force magique susceptible dêtre un antidote à la malchance ou aux maléfices. Dans les pratiques rituelles des populations gabonaises, de manière générale, ces objets sont souvent des ossements, des peaux, des plumes, des dents d'animaux, des fragments de racines d'arbres et autres objets hétéroclites (Raponda-Walker et Sillans I 983:77).

Ils ont pour but de porter du bonheur ou de communiquer un pouvoir surnaturel. D'ailleurs, ils tirent leur valeur du système de correspondance fondé sur des liens de sympathie ou d'antinomie entre leurs éléments et les périls qu'ils sont censés éloigner des humains (Camus, 2001:78).

Ainsi, pour bien être et se sentir à leur poste de travail, de nombreux usagers du bureau déploient un certain nombre d'astuces en abritant des objets divers dans leur espace de travail afin de préserver leurs acquis. Mais à côté de ces efforts que l'on pourrait qualifier d'irrationnels, ces mêmes employés font faire valoir d'autres atouts moins évidents pour les esprits rationalistes.

Alors, que dire de plus sur le fétiche au bureau sinon qu'il est une expression de la modernité.

N'est-ce pas là aussi la conséquence du malentendu civilisationnel de la notion de pouvoir théorisé par Max Weber (1995), développé et mis en pratique par les colonisateurs pendant tant de décennies?

Les deux conceptions de l'usage du bureau comme espace public nous inspirent la question de la gestion privée, personnelle ou informelle d'un espace public. En nous basant sur la gestion du bureau comme d'un espace public, n'est-ce pas là une confiscation de cet espace ? Un domaine que l'on emmène avec soi lorsqu'on est obligé de quitter le bureau ${ }^{23}$.

Le fétiche est une pratique universelle qui s'adapte à chacun des environnements dans lesquels il est pratiqué et par conséquent, répond à une exigence symbolique propre à toute société. Comme le souligne G. Balandier (I954:28I), en situant la société actuelle entre deux époques, le recours à des formes socioculturelles anciennes, pour satisfaire des fins radicalement nouvelles, s'impose, en partie, pour cette même raison. Conserver une façade devant laquelle le colonisateur s'était toujours trouvé désarmé.

En effet, si les formes classiques d'appropriation du bureau sont universelles à travers les expressions de celles-ci, le fétiche, au Gabon repose, quant à lui, sur deux aspects liés au fonds culturel africain de manière générale. Le fétiche se déroule encore dans les villages pour bénéficier de la grâce des esprits.

23 Les exemples sont légion de ceux qui partent leur bureau en emportant tout ou presque. 
La pratique villageoise, à travers l'usage des fétiches, a été transposée dans un environnement urbain dans le cas d'espèce en modifiant ainsi le bureau comme lieu d'exercice du pouvoir public en lieu de cristallisation de toutes les défenses et protections inspirées de la société.

Finalement, les sociétés ne sont jamais ce qu'elles paraissent être ou ce qu'elles prétendent être. Elles doivent en conséquence, être considérées à deux niveaux au moins : l'un superficiel, présente les structures “officielles", si l'on peut dire, l'autre profond, permet d'accéder à la fois aux rapports réels plus fondamentaux et aux pratiques révélatrices de la dynamique du système social environnant. Au fond, pour paraphraser J. Goetz (Thomas et Luneau I 995: I 5), ce qui diversifie les civilisations, c'est justement la manière propre à chacune d'elles, d'aborder la réalité et d'établir ses catégories car un monde "étranger" est toujours plus complexe qu'il n'y paraît au premier abord et chacun des éléments qui le constituent, chacune des valeurs qui le justifient demandent, pour être véritablement compris, à être situés dans l'ensemble socioculturel auquel ils se réfèrent et en dehors duquel ils n'ont plus de signification.

\section{Bibliographie}

Akoun A. et Ansart P., 1999, Dictionnaire de Sociologie, Paris, Le Robert, Seuil.

Hampâté- Bâ Amadou, 1994, Oui mon commandant!, Mémoires II, Arles, Actes Sud, , $399 \mathrm{p}$.

Balandier Georges, 1965, "Problématique des classes sociales en Afrique noire », Cahiers Internationaux de Sociologie, (XXXVIII-196) : 131-142

Balandier Georges, 1954, "Les problèmes du travailleur africain au Gabon et au Congo", Bulletin International des Sciences Sociales, (3) : 505-517.

Balandier Georges, 1982, Sociologie actuelle de l'Afrique noire, Paris, PUF, 4è éd., 530 p.

Balandier Georges, 1957, Afrique ambiguë, Paris, Plon, Coll. 10/18, , 313 p.

Bernault Florence et Tonda Joseph, 2000, "Dynamiques de l'invisible », Politique Africaine, (79) : 5-16

Bidet Alexandra, Pillon Thierry et Vatin François, 2000, Sociologie du travail, Paris, Montchrestien, $437 \mathrm{p}$.

Camus Dominique, 2001, La sorcellerie en France aujourd'bui, Luçon, Editions Ouest-France, $127 \mathrm{p}$.

Castoriadis Cornelius, 1975, L'institution imaginaire de la société, Paris, Seuil, 4è 
éd., 502 p.

Corten A. et Mary André, 2000, Imaginaires politiques et pentecôtismes. Afrique/ Amérique latine, Paris, Karthala, 368 p.

Crozier Michel, 1963, Le phénomène bureaucratique, Paris, Seuil, 382 p.

Durand Gilbert, 1992, Structures anthropologiques de l'imaginaire, Paris, Dunod, $11^{\text {ème }}$ éd., $536 \mathrm{p}$.

Durand Gilbert, 1993, L'imagination symbolique, Paris, PUF, 3è édition, 133 p. Erny Pierre, 1999, Ecoliers d'bier en Afrique centrale, Paris, l'Harmattan, 287 p. Fassin Didier, 1992, Pouvoir et Maladie en Afrique, Paris, PUF, 359 p.

Favret-Saada, J. 1977, Les mots, la mort, les sorts, Paris, Gallimard, 427 p. Fischer Gustave-Nicolas, 1983, Le travail et son espace, Paris, Bordas, 95 p. Fischer Gustave-Nicolas, 1989, Psychologie des espaces de travail, Paris, A. Colin, 217 p.

Gaulejac V. De et al..., 1994, La lutte des places. Insertion et désinsertion, Paris, Marseille, Desclée de Brower, Hommes et perspectives, 286 p.

Gaurier Dominique et Hesse Philippe-Jean, 1987, «LE SCEAU, LA PLUME, LE SEING ET LE NEUD ou l'expression de la volonté individuelle dans les actes juridiques " Textes et Langages XIV - InTERsignes NanTAIs, Université de Nantes, pp 67-78.

Geschiere Peter, 2000, "Sorcellerie et Modernité: retour sur une étrange complicité », Politique Africaine, (79) :17-32.

Geschiere Peter, 1995, Sorcellerie en Afrique. La viande des autres, Paris, Karthala, 302 p.

Gresle François, Perrin Michel,PanoffMichel et Tripier Pierre,1990,Dictionnaire de Sciences Humaines; Sociologie, Psychologie sociale, Anthropologie, Paris, Nathan.

Goody Jack, 1994, Entre l'oralité et l'écriture, Paris, PUF, 323 p.

Javeau Claude, 1991, La société au jour le jour. Ecrits sur la vie quotidienne, Bruxelles, De Boeck Université, 292 p.

Laburthe-Tolra, Philippe et Falgayrettes-Leveau, Christiane, 1991, Fang, Paris, Dapper, 324 p.

Laburthe-Tolra Philippe, 1985, Initiations et sociétés secrètes au Cameroun. Essai sur la religion beti, Paris, Karthala, , 440 p.

Lazorthes Guy, 1999, L'imagination. Source d'irréel et d'irrationnel. Puissance c réatrice, Paris, ellipses, $125 \mathrm{p}$.

Mary André, 1987, Sorcellerie bocaïne, sorcellerie africaine. Le social, le symbolique et l'imaginaire, Cahiers du LASA, $\mathrm{n}^{\circ} 7$. Laboratoire de sociologie anthropologique de l'Université de CAEN, $1^{\text {er }}$ semestre, 125-173.

Ndombi Pierre, 1995, Administration publique en Afrique Equatoriale Française (AEF), 1920-1956. Histoire d'un Etat providence colonial?, Thèse de 
Doctorat en Histoire, Université de Provence, Aix-Marseille I, 3 vol., 1078 p.

Ngoua-Nguéma Ignace, 1998, La fonctionnalité de deux mouvements religieux au Gabon: Béthanie et le Christianisme céleste, $\mathrm{Ph}$. D de sociologie, Université Laval, Québec, 355 p.

Pélegrin-Genel Elisabeth, 1994, L'angoisse de la plante verte sur le coin du bureau, Paris, ESF éditeur, $151 \mathrm{p}$.

Perrois Louis, 1997, L'esprit de la forêt. Terres du Gabon, Bordeaux, 237 p.

Pouillon Jean, 1975, Fétiches sans fétichisme, Paris, François Maspero, $351 \mathrm{p}$.

Raponda-Walker André et Sillans Roger,1983, Rites et croyances des peuples du Gabon, Paris, Présence Africaine/ ACCT, 377p.

Rossatanga-Rignault Guy, 1999, Des "missiles kappa » et des "hommespanthères "... A propos du droit et du surnaturel au Gabon, $\underline{\text { Hebdo }}$ information, (401) : 89-100.

Sow Ibrahima, 1978, Les structures anthropologiques de la folie en Afrique noire, Paris, Payot, N.p.

Surgy Albert (de), 1993, Fétiches II. Puissance des objets. Charme des mots, Paris, Ecole Pratique des Hautes Etudes, 233p.

Surgy Albert (de), 1995, La voie des fétiches. Essai sur le fondement théorique et la perspective mystique des pratiques des fétiches, Paris, L'Harmattan, 441p.

Tarot Camille, 1999, De Durkheim à Mauss, l'invention du symbolisme : sociologie et science des religions, Paris, éditions La Découverte et Syros, 718p.

Thomas Joël, 1998, Introduction aux méthodologies de l'imaginaire, Paris, ellipses, $319 \mathrm{p}$.

Thomas Louis-Vincent et Luneau René, 1995, La terre africaine et ses religions, Paris, l'Harmattan, $233 \mathrm{p}$.

Tonda Joseph, 2000, "Capital Sorcier et Travail de Dieu », Politique Africaine, (79) : 48-65

Tonda Joseph, 2002, "Economie des miracles et dynamiques de subjectivation/ civilisation en Afrique Centrale ", Politique Africaine, (87) : 20-44.

Weber Max, 1995, Economie et Société/1, Les catégories de la Sociologie, Paris, Plon , $410 \mathrm{p}$. 